\title{
Microfabricated Platform for Nanoscale Flow Sensing and Control
}

\author{
Jason Shih and Yu-Chong Tai \\ Electrical Engineering \\ California Institute of Technology \\ Pasadena, CA, USA \\ Email: jshih@mems.caltech.edu
}

\author{
Yunan Miao and Terry D. Lee \\ Immunology \\ Beckman Research Institute \\ Duarte, CA USA
}

\begin{abstract}
In this paper we describe a microfabricated platform for nanoscale flow sensing and control. Two custombuilt pneumatically-driven pumps were used to deliver two different fluids into our microfluidic chip. The two liquids were mixed on-chip and the flow rate and composition of the resulting liquid was measured. Flow rate measurements were accurate within a few $\mathrm{nL} / \mathrm{min}$ and the composition within $0.2 \%$. In this paper, composition is defined as the makeup (by volume and expressed as a percentage) of a binary mixture. Feedback from the sensors was used to control the pumps in order to produce the desired flow profiles.
\end{abstract}

\section{INTRODUCTION}

Flow sensors and sensors which measure the composition of a fluid are well described in literature [1]. Unfortunately, to this date, there has been little actual application of these developed sensors in lab-on-a-chip systems. Sensors can not only make analysis systems more robust, they also enable more complicated fluidic tasks to be done on-chip. One barrier that prevents the integration of sensors is incompatible fabrication processes. Sensors made using one fabrication process may be difficult, if not impossible, to integrate into systems fabricated using a different technology. Another problem is that many of the demonstrated sensors are not universal. For example, flow sensors may only work with a particular kind of fluid or only in a certain flow rate range. These limitations further reduce the number of applications a particular sensor can be used in.

The target application for our flow control platform is liquid chromatography (LC). LC is one of the most powerful and universal techniques for separating and analyzing complex mixtures. But it has very strict requirements regarding flow stability and control. A commercial state-ofthe-art binary nano-LC pump is designed to deliver flows as low as $100 \mathrm{~nL} / \mathrm{min}$ with accurate control over the liquid composition. Precise control is necessary for optimal and repeatable separations. Previously, we demonstrated a fullyintegrated LC system on a chip [2]. But we found that performance and repeatability suffers without any feedback system in place. Only with sensors can we hope to replicate the kind of reliability seen in commercial LC systems.

Depending on the type of stationary phase and mobile phase selected, LC can be used to separate a sample based on size, charge, hydrophobicity, and other molecular properties. We are focusing on reverse-phase (RP) gradient LC which is the backbone of most proteomic analysis. In this type of separation, the sample is first loaded onto a hydrophobic stationary phase. Once the sample is loaded onto the column, a gradient, in which the organic component (generally a non-polar solvent like Acetonitrile, with the other component being water) of the eluent is increased over time, is passed through the column. As the organic component is increased, more and more hydrophobic analytes are eluted off the column. As a result, the sample is separated based on hydrophobicity. A typical gradient might go from 0 to $30 \%$ Acetonitrile at a rate of $1 \% / \mathrm{min}$, all the while maintaining a constant flow rate of $150 \mathrm{~nL} / \mathrm{min}$. Our calibrations and experiments reflect these guidelines.

There are several factors that complicate the development of sensors for use in an LC system. First of all, separations are conducted at very high pressure, sometimes higher than 100 bars. Not only is the pressure high, but the backpressure generated can vary over 1-2 orders of magnitude. The sensors used in this application must be able to withstand these pressures as well as maintain stability over the entire range. A second difficulty is that the composition of the fluid can change over time, as will be the case for our proposed separations. Over the course of a separation, the electrical and physical properties of the fluid will change drastically. Because of this, only sensors which are immune to fluctuations in these fluid properties are viable.

There are many reasons why we are targeting a chipbased LC solution with integrated sensors. In the simplest scenario, the sensors can be used to monitor the exact flow conditions (delivered via a commercial pump) in the column. This can not only help troubleshoot problems, but could also prove valuable in comparing separations done using different LC systems. More importantly, the sensors can also be used

This research was funded in part by the National Institutes of Health. 
for feedback. By having the sensors positioned between the mixer and the column, the column conditions can be monitored and varied in real time with minimal delay. This is in comparison to modern LC pumps which typically have a solvent delay of 10 minutes or more. The ability to control eluent composition on the fly can reduce analysis times and should also theoretically allow us to produce more repeatable separations. This work specifically serves to demonstrate the importance of sensors to on-chip LC. We, however, believe that the importance of on-chip sensors is generic, especially for the lab-on-a-chip field.

\section{DeVICE Design}

There are four components to the presented system: the pneumatically-driven pumps, the mixer, the composition sensor, and the flow sensor. The last three components are all on the microfluidic chip.

\section{A. Mixer}

We designed a completely passive mixer based on diffusion. The two incoming flows were directed into a long narrow channel $(30 \mu \mathrm{m} \times 25 \mu \mathrm{m} \times 8.7 \mathrm{~mm}(\mathrm{WxHxL}))$. The narrow cross section reduced the distance molecules had to diffuse for complete mixing. Even at flows up to several hundred $\mathrm{nL} / \mathrm{min}$, the dwell time in the mixer was sufficient to ensure mixing.

\section{B. Composition Sensor}

The composition of the fluid was determined using a measurement of the electrical properties of the fluid. By measuring the impedance of the fluid being output at the mixer, the exact fluid composition could be deduced. This sensor is shown in Fig. la.

\section{Flow Sensor}

Because of the previously mentioned requirements, many common types of flow sensors were ruled out. Sensors based on the thermal properties of the fluid were ruled out since the composition of the fluid would be constantly changing. While the sensor could be calibrated for all possible fluid compositions, this was something we wished to avoid. Another common type of flow sensor, based on the pressure drop across a restriction, was also ruled out since the fluid viscosity can change by as much as a factor of two during our separations. Eventually we decided on a time-offlight (TOF) flow sensor. By measuring the TOF of a pulse over a pre-determined distance, the flow rate can be deduced. The advantage of this type of flow sensor is that the sensor readings should be largely independent of the fluid type.

We adopted a flow sensor which measured the TOF of an ion pulse. The design is shown in Fig. 1b. Pulses were produced by applying a short, high voltage square pulse to electrode 1 . This produced an excess and depletion of $\mathrm{H}^{+}$ ions at electrodes 1 and 2 respectively. These ion pulses then traveled downstream and were detected by measuring the impedance of the fluid across electrodes 3 and 4 . Our flow
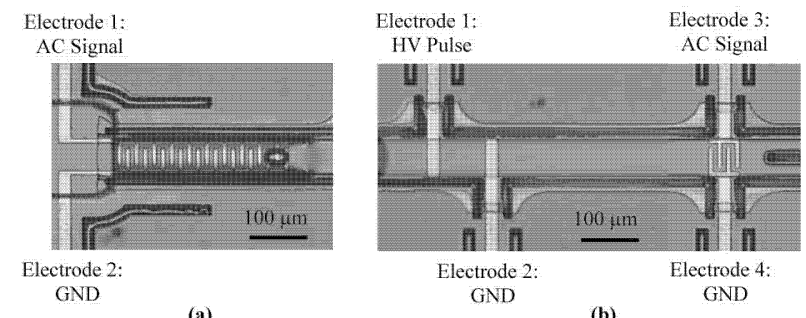

Fig. 1. Layout of the described sensors. (a) Composition sensor. Channel cross section was $80 \mu \mathrm{m} \times 25 \mu \mathrm{m}(\mathrm{WxH})$. Sensor length was $300 \mu \mathrm{m}$. (b) TOF flow sensor. Fluid flow was from left to right. Channel cross section was $80 \mu \mathrm{m} \times 4 \mu \mathrm{m}(\mathrm{WxH})$. The flight path from electrode 1 to the detector electrodes was $500 \mu \mathrm{m}$.

sensor was designed to measure flows below $200 \mathrm{~nL} / \mathrm{min}$ and the channel dimensions and electrode spacing were chosen to reflect this. Theoretically, a flow rate of $150 \mathrm{~nL} / \mathrm{min}$ should yield a TOF (generated from electrode 1) of $64 \mathrm{~ms}$.

\section{DEVICE FABRICATION}

These chips were batch fabricated on a silicon wafer using our parylene surface micromachining technology. This single process was able to fabricate all the sensors as well as the fluidic plumbing needed for an LC system. By having a single process capable of fabricating many different individual devices, integration was fairly straightforward.

The fabrication process started with the growing and patterning of $4000 \AA$ oxide layer on a silicon wafer. Next, a $2000 / 200 \AA \mathrm{Pt} / \mathrm{Ti}$ layer was deposited and patterned on the wafer. This single metal layer was used to define the sensors as well as the metal leads and pads for electrical connections. Next, a sacrificial photoresist layer was spun on the wafer. By using two exposures, one full, and one partial, a bi-level $(25 \mu \mathrm{m} / 4 \mu \mathrm{m})$ photoresist structure was produced. A bi-level sacrificial layer was necessary to reduce the channel cross section where the flow sensor was located. Reducing the cross-section was necessary to ensure a fast linear pulse velocity, which allowed us to design our sensor with a longer flight path. Before parylene was deposited to encapsulate the channels, a DRIE process was used to etch $40 \mu \mathrm{m}$ deep trenches on the sides of the channels. These trenches served to anchor the subsequent parylene layer to the substrate. By mechanically anchoring the encapsulating layer, much higher pressures could be sustained by the channels. After a $10 \mu \mathrm{m}$ parylene layer was deposited, a thick photoresist layer was spun on the wafer and patterned. This photoresist layer was used as a mask to etch shallow $30 \mu \mathrm{m}$ trenches using DRIE. These etched trenches were used to anchor the SU-8 layer onto the substrate. This helped prevent SU-8 delamination during prolonged contact with organic solvents. After the trenches were etched, an $\mathrm{Al}$ mask was used to etch the parylene layer to open the ends of the channels. Finally, a 50 $\mu \mathrm{m}$ SU-8 layer was spun on the wafer and patterned. This SU-8 layer helped to further strengthen the channels and also planarized the chip surface, which facilitated chip packaging. The sacrificial photoresist layer was dissolved away leaving the final desired structure. The fabrication process flow is shown in Fig. 2. The completed chip is shown in Fig. 3. 


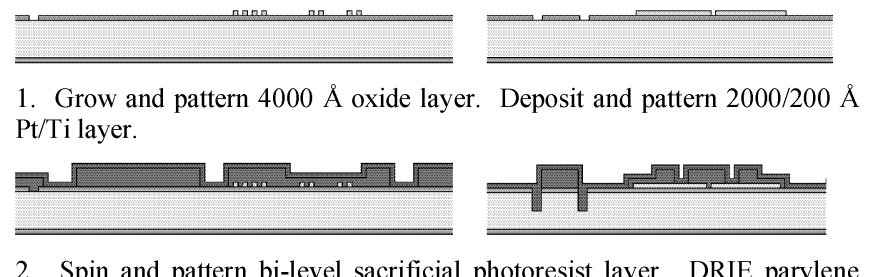

2. Spin and pattern bi-level sacrificial photoresist layer. DRIE parylene anchors. Deposit $10 \mu \mathrm{m}$ Parylene layer.

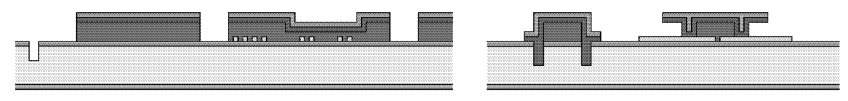

3. DRIE SU-8 anchors. Pattern parylene layer using oxygen plasma and Al etch mask.

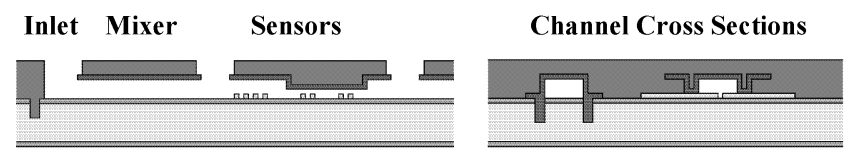

4. Spin and pattern SU-8 layer. Dissolve sacrificial photoresist layer in Acetone.

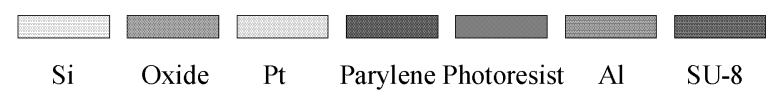

Fig. 2. Process flow for microfluidic chip.

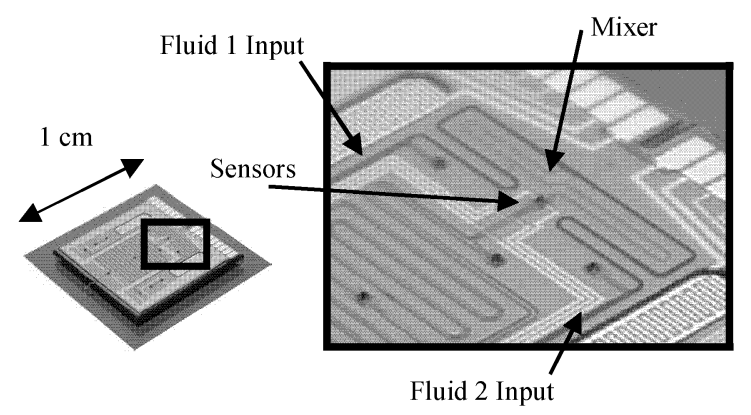

Fig. 3. Picture of the fabricated chip. The portions of the chip relevant to this paper are highlighted.

There were a few key features of the developed process which made it particularly well suited for our targeted application. First of all, we were able to create structures that could withstand pressures exceeding 70 bars for extended periods of time. A second important property of our chips was that they were compatible with the solvents we plan to use. Parylene is well studied as a very inert material. It does not swell in organic solvents nor will it leach impurities which could contribute to noise.

\section{EXPERIMENTAL SETUP}

Pump-to-chip fluidic coupling was accomplished by the use of a custom built manifold. Sealing between the manifold and the chip was provided by o-rings. This manifold was connected to the pump using silica tubing and commercially available high pressure fittings. All electrical connections were done using spring loaded electrical probes

The chip was calibrated by connecting it to a commercial nanoflow HPLC pump. This pump will eventually be the benchmark upon which our system will be compared.
Calibrations were conducted using aqueous solutions of Acetonitrile.

We applied a $+25 \mathrm{~V}, 7$ ms pulse to electrode 1 (refer to Fig. 1b) to generate our ion pulse. Detection was done downstream by applying a $20 \mathrm{kHz}, 100 \mathrm{mV}$ AC signal across electrodes 3 and 4 . The resulting current was measured using an $\mathrm{I} \rightarrow \mathrm{V}$ converter and fed into a lock-in amplifier. Total $\mathrm{I} \rightarrow \mathrm{V}$ gain was $5 \times 10^{7}$. Both the pulse waveform and the waveform of the downstream detector were collected and automatically analyzed. The flow rate was measured at a frequency of $0.1 \mathrm{~Hz}$.

To measure the composition of the fluid, a $500 \mathrm{kHz}, 200$ $\mathrm{mV}$ AC signal was applied across electrodes 1 and 2 (refer to Fig. 1a). The current was measured and converted into a voltage. An AC-RMS conversion was done to turn it into a $\mathrm{DC}$ signal. Total $\mathrm{I} \rightarrow \mathrm{V}$ gain was $5 \times 10^{5}$. One important consideration that had to be taken into account was possible changes in the electrode surface. For example, the samples we plan on analyzing are biological in nature and had been found to adhere to the surface of the electrodes. This caused significant shift in the sensor readings. To negate this effect, the frequency had to be high enough to make the impedance of the double layer capacitance much smaller than the bulk fluid impedance. This approach did have the drawback that if the stray capacitance was too large, the entire circuit would essentially be a short. To overcome this problem, an inductor was placed in parallel with the sensor to produce an LC circuit which resonated at the measurement frequency. This approach is described in detail in [3]. Composition measurements were conducted at $1 \mathrm{~Hz}$.

Once the sensors were properly calibrated, the chip was connected to our custom-built pumping solution. This pump consisted of two electrically controlled pressure regulators which directly regulated the pressure (0-40 bars) in two separate $500 \mu \mathrm{L}$ solvent loops. One loop was filled with a 100/0/0.1 (Water/Acetonitrile/Formic Acid) solution while the other loop was filled with a 40/60/0.1 solution. By combining these two fluids in various ratios, the full range of solvent compositions necessary for a typical RP-LC analysis could be attained. The two pumps were connected directly to the chip via silica tubing. By appropriately varying the pressures in the two solvent reservoirs, the flow rate and composition of the resulting fluid could be varied.

Finally, the flow rate and composition could be accurately controlled by using the sensors for feedback. All sensor functions were controlled via Labview as were the pump control algorithms.

\section{Results AND Discussion}

Fig. 4 shows some sample waveforms from the TOF flow sensor. The valley is from the ion pulse generated at electrode 2 (refer to Fig. 1b) and the peak is from electrode 1. We used the peak for our TOF calculation. For a flow rate of $150 \mathrm{~nL} / \mathrm{min}$ the TOF was measured to be around 67 $\mathrm{ms}$; in close agreement with the theoretical value. We saw variations in the TOF of $+/-0.6 \mathrm{~ms}$ when pumping at a 


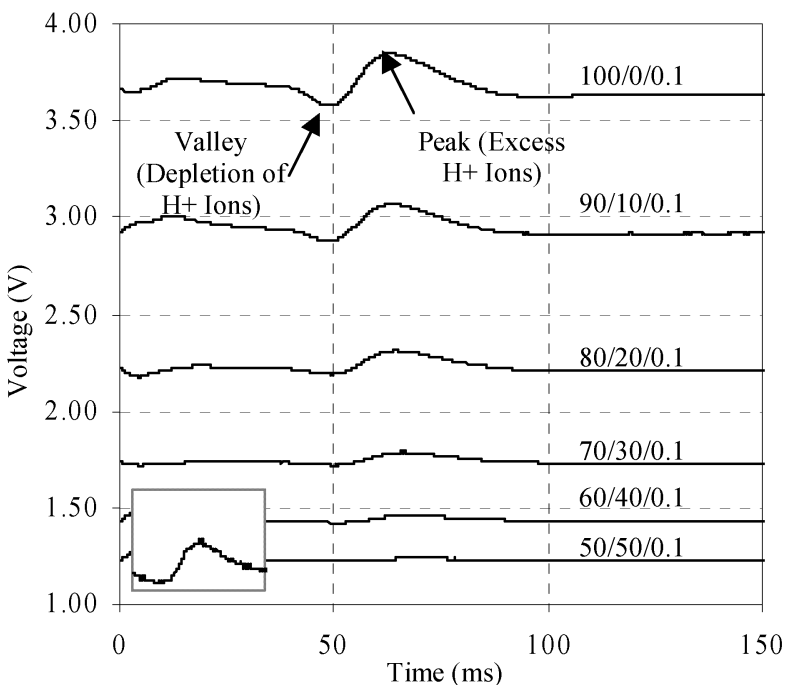

Fig. 4. TOF sensor waveforms for various liquid compositions (expressed a as percentages water/Acetonitrile/Formic Acid). Flow rate was $150 \mathrm{~nL} / \mathrm{min}$ in all cases. The pulse was applied at $\mathrm{t}=0 \mathrm{~ms}$. The inset shows a zoom in of the $50 / 50 / 0.1$ peak.

constant composition and flow rate. This translated to an error of $+/-1.6 \mathrm{~nL} / \mathrm{min}$. Since actual separations are conducted at a constant flow rate, we only calibrated the sensor at the intended operating point. Near this calibrated point, an inverse relationship was assumed. For reference, we also obtained a TOF of $98 \mathrm{~ms}$ at $100 \mathrm{~nL} / \mathrm{min}$. This is good confirmation of the expected inverse relationship. While the waveform shapes did change depending on the solution composition, the peak position was relatively consistent. At the higher organic compositions, there was a slight increase in the TOF by $2-3 \mathrm{~ms}$. This is most likely because electrodes 1 and 2 (refer to Fig. 1b) are close enough that there is some merging of the two ion pulses. As the individual pulses change intensity and shape due to variations in the electrochemical properties of the fluid, the peak positions will shift slightly as well. The shifts in the baseline voltage were expected due to the changing bulk properties of the fluid.

Fig. 5 shows a calibration done for the composition sensor. The composition was varied and the resulting signal was recorded. Sensor readings were accurate within +/$0.013 \mathrm{~V}$ which translates to an error of $0.17 \%$ Acetonitrile. The operation frequency of this sensor was tuned for maximum sensitivity in the 0 to $30 \%$ range. This was done to make the gradient as accurate as possible.

Finally, we demonstrated the use of our on-chip sensors for feedback control. Fig. 6 shows a typical gradient produced using our flow control system. For this demonstration, the output fluid was directed into the on-chip separation column to generate a realistic backpressure. The column was approximately $3 \mathrm{~cm}$ long and packed with $3 \mu \mathrm{m}$ beads. Fluctuations of the flow rate from the desired setpoint were generally less than $5 \mathrm{~nL} / \mathrm{min}$. Fluctuations of the composition were less than $0.3 \%$ Acetonitrile.

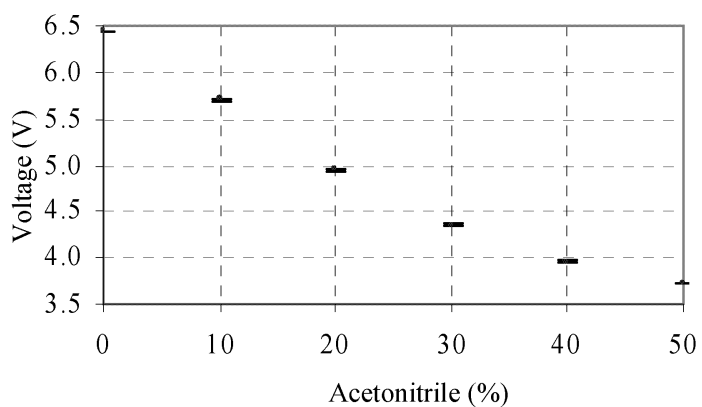

Fig. 5. Calibration curve for the composition sensor. Error bars are all roughly $+/-0.013 \mathrm{~V}$.
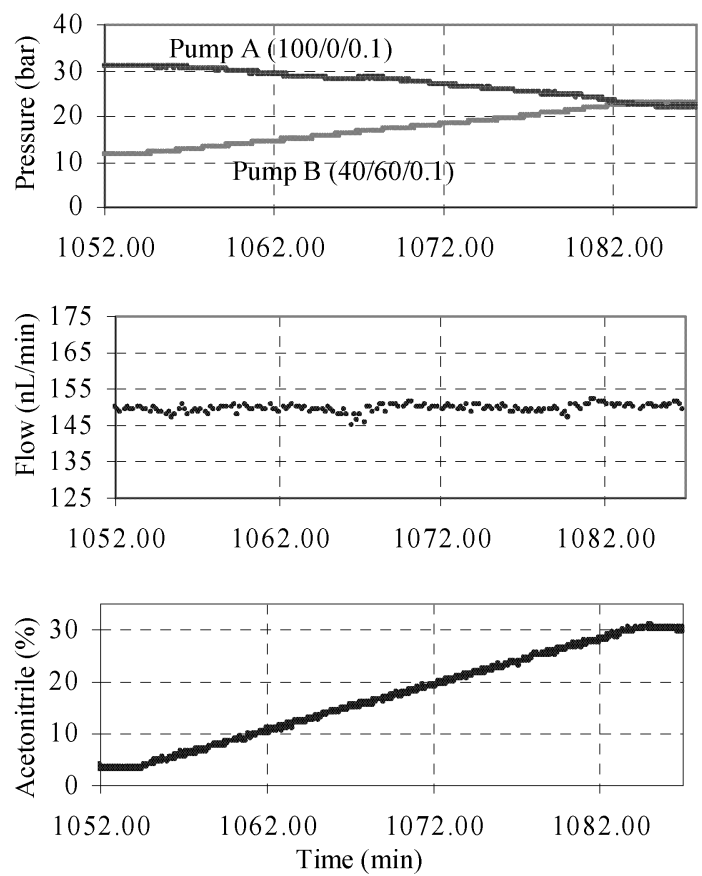

Fig. 6. An example of a linear gradient from $3 \%$ to $30 \%$ Acetonitrile at a flow rate of $150 \mathrm{~nL} / \mathrm{min}$. Shown (from top to bottom) are the pressures for the two pumps, the flow rate, and also the fluid composition.

\section{CONCLUSION}

We demonstrated a feedback system which makes use of our on-chip sensors to accurately control both flow rate and fluid composition. While not discussed here, we have used our feedback system to produce separations nearly identical to those conducted using a commercial HPLC pump, thus proving the effectiveness of our presented platform.

\section{REFERENCES}

[1] G.T. Kovacs, Micromachined Transducers Sourcebook, 1st ed., New York: McGraw-Hill, 1998, pp.687-881.

[2] J. Xie, Y. Miao, J. Shih, Y.C. Tai, and T.D. Lee, "Microfluidic Platform for Liquid Chromatography-Tandem Mass Spectrometry Analyses of Complex Peptide Mixtures," Analytical Chemistry, 77(21), pp. 6947-6953, 2005.

[3] C.Y. Shih, W. Li, S. Zheng, and Y.C. Tai, "A Resonance-Induced Sensitivity Enhancement Method for Conductivity Sensors." Proc. IEEE Sensors 2006, in press 